\title{
Evaluación formativa e interdisciplinariedad: Análisis de dos asignaturas con el mismo sistema de evaluación
}

\author{
Antonio Fraile Aranda \\ Universidad de Valladolid
}

(Received August 14, 2011; Accepted December 23, 2011)

\begin{abstract}
RESUMEN: Este artículo presenta una experiencia basada en el diseño de un mismo sistema de evaluación formativa para dos asignaturas de la formación inicial del profesorado de educación física dentro del grado de Educación Primaria, que se desarrollan de forma conjunta a partir de un tratamiento interdisciplinar. Esto posibilita evaluar en los estudiantes el conocimiento didáctico y el conocimiento disciplinar recogido en ambas asignaturas. Para ello, recogemos y analizamos las ventajas e inconvenientes que señalan los estudiantes sobre (a) el desarrollo conjunto de esas asignaturas y (b) los criterios establecidos, de forma negociada y colaborativa, entre el profesor y los estudiantes para cada uno de los aspectos a ser evaluados.
\end{abstract}

Palabras clave: evaluación formativa, formación del profesorado, interdisciplinariedad.

Formative assessment and interdisciplinary: Analysis of two subject matters with the same evaluation system

\begin{abstract}
This article presents an experience based on the design of a single system of formative evaluation for two subject matters in an undergraduate teacher education program in physical education in Primary Education, both of which are developed jointly from an cross curricular point of view. This allows evaluating the students in both didactic knowledge and curricular knowledge on both subject matters. For this purpose, we have collected and analyzed the advantages and barriers pointed out by the students as regards to (a) the concurrent development of these subject matters and (b) the criteria established jointly by the professor and the students for each of the aspects to be evaluated.
\end{abstract}

Keywords: formative assessment, teacher training, interdisciplinary.

\section{INTRODUCCIÓN}

Los cambios en la sociedad del conocimiento precisan de experiencias de innovación que ayuden a una mayor cualificación de los estudiantes durante su formación inicial. A través de mejorar la transmisión de los conocimientos y fomentar relaciones democráticas con los estudiantes como futuros docentes y también futuros ciudadanos. Desde unas prácticas democráticas que fomenten la relación entre iguales, eviten las relaciones de poder y establezcan una mejor conexión entre el estudiante, el contenido curricular y el docente. Por ejemplo, 
una de las finalidades del sistema educativo es formar a los futuros docentes en el respeto por la dignidad de los estudiantes (Marina, 1995).

El modelo formativo tradicional, academicista y tecnológico de la enseñanza universitaria se ha venido caracterizando por una fragmentación del conocimiento, un contenido curricular organizado y secuenciado de forma cerrada, un elevado número asignaturas, una desconexión entre las enseñanzas teóricas y prácticas, una escasa colaboración entre el profesorado, así como un sistema de evaluación finalista sobre los resultados de los estudiantes. Por tanto, esa falta de interrelación entre los elementos del programa dificulta la comunicación entre los saberes; a lo que se debe añadir la especialización de las asignaturas y de los docentes que las imparten.

Como alternativa a ese proceso formativo, se debe apostar por una práctica globalizada e interdisciplinar, que conecte la teoría y la práctica, y que convierta a los estudiantes en profesores reflexivos (Dewey, 1989; Schön, 1992). Aprender a revisar su práctica docente, de forma retrospectiva, y a utilizar sus vivencias escolares como medio para reconstruir sus conocimientos. También y de acuerdo con Johnson \& Johnson (1999, 2009), dicha práctica docente debe partir de un aprendizaje colaborativo porque ayude a los estudiantes a aprender juntos, para resolver mejor los problemas y compartir sus significados. Por último, el uso de una enseñanza activa y participativa facilita que los estudiantes aprendan de forma más comprensiva y menos competitiva.

Una larga tradición pedagógica ha venido resaltando la importancia de organizar y ordenar los programas formativos en torno a diseños globales, con objeto de conseguir una mayor integración de sus contenidos. Según Torres (1996), la organización interdisciplinar entre materias favorece un enriquecimiento mutuo, ayudando a la elaboración de marcos conceptuales más generales en los que diferentes materias se ven implicadas y modificadas, permitiendo pasar a depender unas de otras. Igualmente, Gimeno Sacristán (1988) considera que la agrupación de contenidos, desde una perspectiva interdisciplinar, favorece la motivación de los estudiantes ya que aprecian una mayor coherencia entre los contenidos, las relaciones entre contenidos diversos, la conexión entre actividades teóricas y prácticas, la ordenación del trabajo en el aula y en los propios centros, el establecimiento de ciclos de actividades para contenidos con una coherencia interna y, por último, la unidad entre tópicos y materias.

Por tanto, con objeto de satisfacer con éxito las exigencias complejas del sistema educativo, de acuerdo con Rychen \& Salganik (2006), un tratamiento interdisciplinar favorecerá un desarrollo más globalizado de las competencias y, con ello, podremos integrar dentro del proceso de enseñanza-aprendizaje los conocimientos, las habilidades y destrezas, las actitudes, las emociones y los valores.

En este ámbito de la educación superior, se han venido desarrollando algunas experiencias de coordinación entre asignaturas para un mismo sistema de evaluación. Su finalidad era que el alumnado fuera capaz de transferir conocimientos entre diferentes asignaturas, de la misma área disciplinar, y de cara a conseguir la resolución de una situación educativa real (Santos Pastor, Martínez y López 
Pastor, 2009; López Pastor, 2009).

A partir de estas premisas, comenzamos una experiencia docente con estudiantes de magisterio sobre cómo programar y evaluar dos asignaturas desde un mismo sistema de evaluación formativa. Para ello, se parte de un diseño interdisciplinar formado por dos asignaturas que, al pertenecer al mismo bloque formativo, se favorece una mejor comprensión, globalización e integración de sus contenidos tanto en el ámbito didáctico como en el disciplinar. Esto permite organizar de forma más eficiente la enseñanza y, con ello, el aprendizaje entre los estudiantes. En un estudio reciente (Fraile, 2009), un grupo de estudiantes de educación física señalan la importancia que para su formación inicial ha supuesto la adquisición de algunas competencias transversales, gracias a ese tratamiento coordinado entre las materias. Entre ellas, destacan la mejora de su desarrollo personal y, de manera especial, el trabajo en equipo y el desarrollo de los contenidos desde una perspectiva interdisciplinar.

Con objeto de presentar en este artículo dicha experiencia formativa, pasaremos a describir el contexto en donde se ha desarrollado, para a continuación presentar la propuesta sobre un sistema de evaluación conjunto para esas dos asignaturas a través de una revisión de competencias docentes. Por último, se analizarán las dificultades y ventajas que ha supuesto ese tratamiento interdisciplinar.

\section{CONTEXTO Y DESARROLLO DE LA EXPERIENCIA}

Esta tarea docente se realiza con estudiantes de magisterio de la especialidad de Educación Física, desde las asignaturas de Educación Física y su Didáctica (nueve créditos) y Educación Física en Educación Primaria (seis créditos). $\mathrm{Su}$ implementación se ha llevado a cabo, en los últimos ochos años, en un grupo aproximado de cuarenta estudiantes, dedicando tres sesiones de clase semanales para ambas materias a lo largo de todo el curso.

Para el diseño, puesta en práctica y evaluación del programa se ha venido adoptando un proceso de negociación entre el profesor y los estudiantes. Con ello se trataba de favorecer la colaboración entre todos para acercar mejor ese conocimiento a las características de un grupo multicultural. Entre otras cuestiones claves, en dicho proceso se les preguntó sobre ¿qué esperaban aprender a través de estas materias? ¿cómo se debería organizar el proceso de enseñanzaaprendizaje? ¿cuándo? ¿ por qué? y ¿para qué?

Antes de negociar los componentes del programa, el profesor y los estudiantes compartíamos nuestros compromisos y obligaciones docentes-discentes. Por tanto: las finalidades, los contenidos, la metodología y los criterios para evaluar se establecían desde una dinámica colaborativa. Con objeto de apoyar este proceso formativo, contamos con la presencia de un observador externo que venía realizando un seguimiento de toda esa actividad docente tanto de los estudiantes como del profesor. Como conclusión de este plan de acción, los estudiantes participaron en el diseño, aplicación y revisión del programa formativo, gracias a: 
a) implicarse de forma crítica y creativa en las diferentes fases y componentes del programa; b) organizar los recursos y materiales disponibles; c) estructurar el espacio y los tiempos de las sesiones y las actividades; y d) desarrollar un aprendizaje colaborativo en donde el profesor también asumía el papel de aprendiz (Altava y Gallardo, 2004; Fraile, 2006).

Esta negociación también exigió de un compromiso ético y de responsabilidad de cada uno de los participantes (profesor, observador externo y estudiantes) recogiendo los siguientes aspectos: a) participar en las actividades de aprendizaje antes, durante y después de clase; b) respetar a los compañeros; c) realizar las lecturas asignadas; y d) escribir en el cuaderno de campo. A través de este aprendizaje colaborativo se pudieron definir los objetivos y las actividades, a través de un espíritu crítico y de una reflexión discursiva (Piaget, 1977). La metodología grupal favorece que los estudiantes aprendan a buscar aprendizajes beneficiosos para ellos y para el grupo, procurando que el conocimiento de cada uno repercuta en el resto (Johnson, Johnson y Holubec, 1999). Como ejemplo, en la presentación de los trabajos en grupo no se decide quien lo hará hasta unos momentos antes y a través de un sorteo; con ello, todos los miembros del grupo deben estar preparados, evitando que intervengan siempre los más cualificados.

A través de la actividad reflexiva, los estudiantes han podido hacer más conscientes las actividades de clase. Esta capacidad cognitiva, según Piaget (1973), se desarrolla a partir de considerar que la acción es el origen del pensamiento y, por tanto, todas las acciones reflexivas ayudan a la construcción del conocimiento. Especialmente en la enseñanza universitaria, debe ser clave la intervención activa de las capacidades intelectuales superiores de los estudiantes, ya que les permite recordar y analizar la acción. En nuestro caso, una vez que los estudiantes describían en su cuaderno de campo lo sucedido durante la clase, analizaban su intervención a nivel cognitivo, motriz y emocional; es decir, se compartían los sentimientos corporales de cada uno de los participantes, para luego buscar aplicaciones didácticas significativas para los escolares de primaria durante el Practicum.

\section{LA EVALUACIÓN FORMATIVA DE LAS COMPETENCIAS}

Al desarrollar este proceso formativo interdisciplinar, uno de los criterios básicos que hemos tenido en cuenta en el diseño del programa ha sido destacar la importante presencia que deben tener las competencias transversales. A través de un modelo educativo globalizado e interdisciplinar hemos podido atender de forma más coherente e íntegra un conjunto de competencias para la formación personal de los futuros docentes.

Por otro lado, según Stobart (2010) es más fácil para los estudiantes aprender algo cuando saben previamente lo que tienen que hacer y adónde quieren llegar. Desde este tipo de evaluación formativa, se promueve un proceso de enseñanzaaprendizaje que pretende hacer más explícitas las finalidades de la evaluación y relacionarlas con los objetivos de las asignaturas. Para ello, nos valdremos de un 
conocimiento basado en principios mediante preguntas y problemas menos previsibles y, por último, buscaremos la manera de conseguir que las calificaciones finales sean más justas.

A continuación, señalaremos en la Tabla 1 cuales son las actividades de evaluación que hemos venido empleando para comprobar la adquisición de competencias por parte de los estudiantes.

Tabla 1. Resumen de Actividades de Aprendizaje e Instrumentos de Evaluación

\begin{tabular}{|c|c|}
\hline Tipo competencias & $\begin{array}{l}\text { Actividades de aprendizaje e instrumentos } \\
\text { de evaluación. }\end{array}$ \\
\hline $\begin{array}{l}\text { Saber } \\
\text { (conocimientos } \\
\text { saberes teóricos) }\end{array}$ & $\begin{array}{l}\text { Informes. Escala de evaluación (Tabla 2) } \\
\text { Prueba escrita. }\end{array}$ \\
\hline $\begin{array}{l}\text { Saber hacer } \\
\text { (procedimientos y } \\
\text { aplicaciones } \\
\text { prácticas) }\end{array}$ & $\begin{array}{l}\text { Informes. Cuadernos de Campo. Escala de } \\
\text { evaluación (Tabla2). Práctica docente con } \\
\text { escolares. Escala de evaluación (Tabla 4) } \\
\text { Asistencia y participación en clase. }\end{array}$ \\
\hline $\begin{array}{l}\text { Ser/saber estar } \\
\text { (desarrollo personal } \\
\text { y actitudinal) }\end{array}$ & $\begin{array}{l}\text { Capacidades socio-morales en el trabajo } \\
\text { grupal (Tabla 5). Presentación de los } \\
\text { informes. Escala de evaluación (Tabla 3) }\end{array}$ \\
\hline
\end{tabular}

a) Competencias relacionadas con los conocimientos y saberes teóricos. Los estudiantes elaboran en grupo un informe de cada uno de los temas que será valorado conjuntamente por el profesor y los miembros del grupo responsable del tema (Fraile, 2004, 2008). Cada uno de los ocho informes recogen los temas del curso y se organizan a partir de diferentes apartados: Se parte de reconocer los conocimientos previos y vivencias de los estudiantes relacionados con el tema. A continuación, los conocimientos adquiridos a partir de las siguientes actividades: lectura de artículos, debates de pequeño y gran grupo, explicaciones del profesor y de los estudiantes que hacen de docentes, esquemas o mapas conceptuales, aplicaciones didácticas, preguntas que emergen de los textos y un glosario con los conceptos y términos "claves" extraídos de los textos. Por último, los estudiantes revisarán de forma crítica el trabajo individual y colectivo del grupo, haciendo propuestas de mejora.

Con objeto de poder evaluar tanto el cuaderno de campo como los informes en la Tabla 2 recogemos los criterios negociados con los estudiantes. 


\section{Tabla 2. Criterios para Evaluar los Informes y los Cuadernos de Campo}

- Recoger cada uno los apartados establecidos para los cuadernos de campo y para los informes.

- Incorporar nuevas propuestas con objeto de ampliar y mejorar los contenidos desarrollados en clase.

- Reflexionar sobre las cuestiones esenciales abordadas en cada uno de los temas.

- Valorar y hacer propuestas de mejora sobre la intervención de cada uno de los grupos.

- Diseñar una propuesta curricular para cada uno de los temas.

Los primeros informes son elaborados por parejas o en pequeños grupos, para luego poder construir cada grupo uno de carácter global. Los cuadernos de campo son el instrumento para que los estudiantes puedan escribir y reflexionar sobre lo tratado en clase de cada uno de los temas. Los informes finales se presentarán por un miembro elegido al azar de cada grupo, siendo coevaluados con una escala numérica de uno a cinco, a partir de unos criterios establecidos por los estudiantes y que se recogen en la tabla 3.

\section{Tabla 3. Criterios para Evaluar las Presentaciones de los Informes Finales}

- Capacidad para la expresión y comunicación verbal y corporal.

- Coherencia entre las aportaciones y argumentos presentados en la exposición (referentes teóricos, vínculos, ejemplos).

- Calidad de los contenidos (que estén presentes en cada uno de los apartados del informe).

- Capacidad para responder a las preguntas del profesor y de los compañeros.

- Capacidad para adoptar una postura de respeto y autocrítica antes las opiniones de los compañeros y las del propio profesor.

Otra de las actividades a evaluar es el conocimiento adquirido por los estudiantes. Para ello, éstos deben elaborar preguntas sobre cada uno de los temas de estudio. Posteriormente, el profesor seleccionará aquellas cuestiones que se consideren más relevantes para el aprendizaje. Para responder, los estudiantes podrán valerse de los materiales escritos durante el curso. Después coevaluarán dichas respuestas. A través de este proceso formativo, todos ellos se implican en la elaboración de las preguntas, en las respuestas y, por último, en su autoevaluación, a partir de los criterios negociados en los grupos.

b) Competencias relacionadas con los procedimientos de saber hacer. Para valorar la capacidad docente, los estudiantes realizarán unas prácticas con un grupo de escolares de primaria. Cada una de las sesiones tendrá una duración aproximada de cincuenta minutos, hasta un total de quince sesiones repartidas entre todos ellos. Mientras que uno dirige la clase, el resto actúan de observa- 
dores a partir de la ficha recogida en la Tabla 4, en la que se contemplan los siguientes aspectos a valorar.

\section{Tabla 4. Aspectos a Observar/Valorar sobre la Práctica Docente de los Estudiantes}

1.- Conoce y tiene en cuenta las necesidades e intereses de los alumnos, así como los factores contextuales (socio-culturales).
a) Recoge los intereses de los escolares vinculados con la práctica.
b) Diseña la clase a partir de la edad y el contexto socio-cultural de los escolares.
c) Considera los conocimientos previos de los escolares.
d) Utiliza un lenguaje adecuado y comprensible al dirigirse a los escolares.

2.- Diseña y pone en práctica las actividades en función de los contenidos, competencias y diversidad de los alumnos.
a) Diseña las actividades considerando las competencias de los escolares.
b) Diseña actividades según los contenidos del tema asignado.
c) Aplica las actividades de forma correcta.
d) Considera la diversidad de edad y conocimientos de los escolares.

3.- Aplica estrategias según las finalidades y contenidos del tema.

a) Aplica estrategias de enseñanza coherentes con las finalidades y los contenidos a enseñar.

b) Comunica e interactúa con los escolares.

c) Identifica los problemas de aprendizaje de los escolares durante la clase.

d) Responde a los problemas detectados.

4.- Establece los criterios, los instrumentos y las técnicas para valorar la participación y el aprendizaje de los escolares, y sobre su propia práctica.

a) Selecciona los criterios para evaluar la práctica de los escolares.

b) Utiliza los instrumentos y técnicas según los criterios anteriores.

c) Informa a los escolares sobre su aprendizaje durante la clase.

d) Reconoce los aciertos y los errores cometidos durante su práctica docente.

Después de la clase, el profesor y estudiantes comentarán y valorarán la práctica de cada uno de ellos. Por último, cada uno de esos estudiantes expondrá su autoevaluación.

c) Competencias relacionadas con los aspectos actitudinales y del saber estar. En este caso, los estudiantes deben revisar en su cuaderno de campo cómo han cumplido con sus compromisos, desde una perspectiva ético-moral, así como analizar cómo ha evolucionado su comportamiento individual y grupal. Es importante conocer de cada uno de los estudiantes: el nivel de participación e implicación en el trabajo grupal tanto durante como después de la clase en el trabajo autónomo del grupo. Para ello, se valoran una serie de competencias transversales relacionadas con la capacidad de diálogo, comunicación, escucha activa y respeto, y otras que recoge Zabalza (2003). Asi como otras, relativas a un compromiso cívico y social con la comunidad educativa según establece Rogers (1972) como son: la toma de decisiones, la capacidad ética y la capacidad crítica. A continuación en la Tabla 5, se presentan algunos aspectos grupales que se encargan los propios estudiantes de coevaluar. 


\section{Tabla 5. Capacidades Socio-morales del Trabajo Grupal}

- Identifica y participa de las cuestiones problemáticas que surgen en el proceso de enseñanza-aprendizaje en el grupo de trabajo.

- Analiza y da soluciones de forma ética y responsable a los problemas surgidos.

- Valora y cuestiona de forma crítica el proceso de aprendizaje colaborativo desarrollado entre el profesor y los estudiantes.

- Reconoce y valora las relaciones socio-morales del profesor con el grupo y de los estudiantes entre sí de: respeto, colaboración, solidaridad e igualdad de oportunidades.

- Identifica y valora el desarrollo de las competencias profesionales vinculadas a su futura actividad profesional: liderazgo, comunicación, empatía, colaboración, resolución de conflictos, etc.

- Justifica de forma crítica las decisones tomadas relativas a la autoevaluación.

- Reflexiona sobre su participación en el proceso de aprendizaje.

- Valora el grado de compromiso alcanzado dentro y fuera de la actividad académica ante situaciones o problemáticas de tipo social.

A partir de esos criterios, los estudiantes han valorado algunas de las tareas de sus compañeros dentro y fuera del aula. A partir de esta evaluación interna, realizada en diferentes momentos del curso, los estudiantes han elaborado un informe justificado de autoevaluación/autocalificación (Fernández Balboa, 2005; Fraile, 2004, 2006; Fraile \& Aragón, 2003; López Pastor, 2004). De acuerdo con los criterios que señala Bain (2006), los estudiantes recogían en su informe final cómo durante el curso han evolucionado sus conocimientos y creencias personales; qué estrategias han utilizado durante el aprendizaje colaborativo; cómo y de dónde han extraído sus conocimientos; qué dificultades han tenido en los procesos de coevaluación y autoevaluación, etc. Por último, para la calificación final los estudiantes tuvieron en cuenta los valores asignados a cada una de las actividades de aprendizaje recogidas en las tablas anteriores.

La falta de experiencia en la organización de los contenidos, así como en el desarrollo de los procesos de evaluación/calificación, ha supuesto que inicialmente algunos estudiantes señalaran algunos inconvenientes, aunque posteriormente valoraran también las ventajas de poder participar de forma activa en este proceso de enseñanza aprendizaje.

\section{INCONVENIENTES DE IMPARTIR DOS ASIGNATURAS DE FORMA CONJUNTA}

Al finalizar el curso, se solicitó a los estudiantes una valoración sobre dicha propuesta, encontrándonos con algunas críticas:

a) Los estudiantes no distinguen bien entre los contenidos de las dos materias que son objeto de estudio. Al comienzo del curso, los estudiantes reconocen que esta agrupación de asignaturas les resulta más compleja que la organización 
tradicional, en donde cada bloque de contenidos es asignado a cada una de las materias. Así, cuando se les preguntaba sobre qué tipo de conocimientos esperaban aprender, no distinguían bien cuáles pertenecían a una u otra asignatura, debiendo consultar la guía didáctica. Ya que los estudiantes cuando se matriculan, no revisan los descriptores de las asignaturas. Más tarde, al tener éstos que participar en el diseño, aplicación y evaluación del programa se cuestionan lo siguiente: ¿Cómo estas dos asignaturas les ayudará a lo largo de su formación? ¿Qué competencias profesionales se desarrollan a través de dichas materias? ¿Cómo se evaluarán los conocimientos y las competencias adquiridas? Según avanza el curso, los estudiantes aprenden a conectar la teoría con la práctica y, con ello, a agrupar ambas asignaturas. Por último, al finalizar el curso la mayoría de los estudiantes valoran de forma positiva dicho tratamiento interdisciplinar, ya que les ha permitido alcanzar una visión más global e integral de los conocimientos, así como participar en un proceso evaluativo más simplificado.

b) Mayor exigencia cognitiva desde los aprendizajes globalizados e interdisciplinares. Los estudiantes reconocen que este modelo de enseñanza-aprendizaje, para integrar dichos contenidos, les exige una mayor capacidad cognitiva. Durante el curso, al conectar ambas materias se consigue una visión más global e interdisciplinar. Los estudiantes precisan de una buena capacidad de análisis y síntesis para buscar la información, así como para construir y redactar los informes. Igualmente, de la capacidad de valoración cuando intervienen en los procesos de coevaluación y autoevaluación, a través de aplicar de forma autónoma y responsable los criterios negociados con el profesor. Por último, esta mejora cognitiva viene determinada al establecer la relación entre la teoría y la práctica de esas dos asignaturas.

c) Dificultades en buscar aplicaciones didácticas al conocimiento teórico. La formación academicista, que viene primando en el escenario universitario, no sólo determina que el conocimiento teórico sea prioritario sobre las aplicaciones didácticas, sino una importante fragmentación del conocimiento. Los estudiantes no están habituados a conectar los conocimientos de ambas asignaturas: La primera (didáctica de la educación física) que recoge la formación teórico-didáctica a través de los objetivos, contenidos, actividades, metodología de enseñanza, evaluación. La segunda (educación física en primaria) con esos mismos contenidos aplicados para la educación primaria. Por tanto, éstos deben adquirir un conocimiento teórico que les ayude a intervenir en la práctica. En el caso de que estas dos materias se impartieran por separado, los profesores deberían dedicar un tiempo para llegar a acuerdos tanto en el desarrollo de los contenidos, como en la posterior calificación de los estudiantes. Igualmente, supondría un mayor número de asignaturas en el programa formativo. En nuestro caso, al ser el mismo profesor el que imparte ambas asignaturas evita los problemas de coordinación y de evaluación antes comentados. 


\section{VENTAJAS DE UN TRATAMIENTO INTERDISCIPLINAR}

Del mismo modo que, para algunos estudiantes, presentar esas dos asignaturas de forma conjunta producía algunas dificultades conceptuales, para otros fue ventajoso ya que les permitía:

a) Conocer en la práctica el proceso de enseñanza-aprendizaje desde un tratamiento interdisciplinar. En nuestro caso, al conectar las dos asignaturas, los estudiantes participan de un proceso interdisciplinar, a través de unificar las tareas de enseñanza-aprendizaje. También, al poder relacionar los contenidos procedentes de esas dos materias, los estudiantes desarrollan su capacidad de síntesis, así como diseñan aplicaciones didácticas con las que afrontar algunos problemas o situaciones complejas del aula.

b) Adquirir un desarrollo más coordinado de competencias específicas y transversales. Desde esta experiencia formativa vinculada al área de educación física, los estudiantes desarrollan aquellas competencias específicas relativas a los aspectos didácticos de esta área de conocimientos, así como transversales en donde se atienden a capacidades interpersonales gracias al desarrollo del aprendizaje colaborativo. Durante el curso, que los estudiantes compartan las competencias a través de esas dos asignaturas ha facilitado su desarrollo tanto para su mejora personal, como para aprender a diseñar las competencias básicas para el contexto escolar de Primaria.

c) Ayudar a superar la fragmentación de los saberes. La conexión entre estas dos asignaturas ha facilitado que los estudiantes conecten mejor el conocimiento teórico-práctico de la Didáctica y la acción aplicativa de la Educación Física en Primaria. Desde esa nueva organización de las asignaturas se consigue evitar la habitual fragmentación disciplinar de los contenidos. Según la teoría de la estructura o configuración (Gestalt), tanto los escolares como también los adultos perciben las situaciones por totalidades, mediante una acción recíproca entre el sujeto y los elementos que integran la situación (todo ello configura una estructura). Por ejemplo, una vez que los estudiantes conocen las diferencias entre las metodologías activas y directivas elaboran estrategias prácticas, a través del descubrimiento guiado y el mando directo, que luego son experimentadas con grupos de escolares durante el periodo de prácticas, considerando el desarrollo madurativo de los escolares, los contenidos de la materia, las actividades y los indicadores para la evaluación. Por tanto, gracias a la conexión de estos contenidos facilita que los estudiantes aprendan de forma más globalizada, a través de superar esa fragmentación de los saberes procedente de los modelos de enseñanza tradicional.

d) Unificar el proceso de evaluación y calificación de los aprendizajes desde un modelo formativo activo y participativo. Al posibilitar los procesos de autonomía, se favorece una participación activa y responsable de los estudiantes en los procedimientos de evaluación y calificación para ambas asignaturas. Tanto el uso de la coevalación como la autoevaluación es valorada de forma positiva por 
parte del grupo, ya que les permite una participación más democrática en dicho proceso docente. Para ello, los criterios para la evaluación final se negocian entre el profesor y los estudiantes, con objeto de llegar a obtener una calificación más justa y en la que todos tengan voz. Por todo ello, consideramos que tanto la coevaluación, la autoevaluación y la autocalificación final pueden representar un ejemplo de cómo el profesor favorece un tratamiento digno y de respeto hacia los estudiantes, desde la autocrítica y la responsabilidad de cada uno de ellos, siendo para el propio profesor una parte importante de su aprendizaje.

\section{CONCLUSIONES}

Para finalizar y con objeto de ayudar a otros docentes preocupados por la formación universitaria, señalaremos que gracias a la conexión interdisciplinar de ambas materias los estudiantes han podido alcanzar una visión más globalizada de la actividad docente, desde las diferentes perspectivas (teórico y práctica); así como un desarrollo más coherente del programa, pues ambas materias pertenecen al mismo campo científico (la didáctica de la educación física en primaria) y al mismo bloque de la formación del profesorado. También han logrado una mejor relación disciplinar entre los conocimientos teóricos, facilitado por el uso de la misma metodología. Por último, este enfoque interdiscplinar nos ha ayudado a superar la fragmentación de los contenidos, reduciendo el número de materias del plan estudios y simplificado el sistema de evaluación orientándole hacia un tratamiento más formativo.

\section{REFERENCIAS}

Altava, V. y Gallardo, I. M. (2004). La construcción conjunta del conocimiento desde la vivencia del propio aprendizaje. Investigación en la escuela, 24, $69-78$.

Bain, K. (2006). Lo que hacen los mejores profesores universitarios. Valencia: PUV.

Dewey, J. (1989). Como pensamos: Nueva exposición de la relación entre pensamiento reflexivo y proceso educativo. Barcelona: Paidós.

Fernández-Balboa, J. M. (2005). La autoevaluación como práctica promotora de la democracia y la dignidad. En A. Sicilia y J. M. Fernández-Balboa (Coord.), La otra cara de la educación física: la educación física desde una perspectiva crítica” (pp. 127-156). Barcelona: Inde.

Fraile Aranda, A. (2004). Un cambio democrático en las aulas universitarias: Unas experiencia en la formación del profesorado de Educación Física. Contextos educativos: Revista de Educación, 6-7, 213-234.

Fraile Aranda, A (2006). Cambios en el aula universitaria ante los nuevos retos europeos. Tándem, 20, 57-72.

Fraile Aranda, A. (2008). El aprendizaje cooperativo como metodología para el desarrollo de los ECTs: Una experiencia de formación del profesorado de 
Educación Física. Revista Fuentes, 8, 37-53.

Fraile Aranda, A. (2009). Generic Competences in the Physical Education Teacher Training. Studia Educatio Artis Gymnasticae. 1, 9-17.

Fraile, A y Aragón, A. (2003). La autoevaluación a partir de los compromisos de los estudiantes de educación física. En Navarro, V. y Jiménez, F. (Eds.), XXI Congreso Nacional de Educación Física. Universidad de la Laguna. Tenerife. CD-R.

Gimeno Sacristán, J. (1988). El curriculum: una reflexión sobre la práctica. Madrid: Morata.

Johnson, D. W. y Johnson, R. T. (1999). Aprender juntos y solos. Aprendizaje cooperativo, competitivo e individualista. Buenos Aires: Aique.

Johnson, D. W. y Johnson, R. T. (2009). An Educational Psychology Success Story: Social Interdependence Theory and Cooperative Learning. Educational Researcher, 38(5), 365-379.

Johnson, D., Johnson, R., y Holubec, E. (1999). El aprendizaje cooperativo en el aula. Barcelona: Paidós.

López Pastor, V. M. (2004). Evaluación, calificación, credencialismo y formación inicial del profesorado: efectos y patologías generadas en la enseñanza universitaria. Revista Interuniversitaria de Formación del Profesorado, 51, 221-232. AUFOP. Zaragoza.

López Pastor, V. M. (coord.). (2004). Evaluación formativa y compartida en educación superior. Madrid: Narcea.

Piaget, J. (1973). Psicología y epistemología. Barcelona: Ariel.

Piaget, J. (1977). Psicología y pedagogía. Barcelona: Ariel.

Rychen, D.S. y Salganik, L.H. (2006). Las competencias clave para el bienestar personal, social y económico. Málaga: Aljibe.

Rogers, C. (1972). El proceso de convertirse en persona. Barcelona: Paidós.

Santos Pastor, M. L., Martínez, L. F., y López Pastor, V. L. (2009). La innovación docente en el EEES. Universidad de Almería: Almería.

Schön, D. A. (1992). La formación de profesores reflexivos. Barcelona: Paidós.

Stobart, G. (2010). Tiempos de pruebas: Los usos y abusos de la evaluación. Madrid: Morata.

Torres Santomé, J. (1996). Globalización e interdisciplinariedad: El currículo integrado. Madrid: Morata.

Zabalza, M. A. (2003). Competencias docentes del profesorado universitario. Madrid: Narcea. 